\title{
乌s \\ Development of a high average current rf linac thermionic injector
}

\author{
S. H. Gold, ${ }^{1}$ A. Ting, ${ }^{1}$ V. Jabotinski, ${ }^{2}$ B. Zhou, ${ }^{3}$ and P. Sprangle ${ }^{1}$ \\ ${ }^{1}$ Plasma Physics Division, Naval Research Laboratory, Washington, D.C. 20375, USA \\ ${ }^{2}$ Beam-Wave Research, Inc., Bethesda, Maryland 20814, USA \\ ${ }^{3}$ Research Support Instruments, Inc., Lanham, Maryland 20706, USA
}

(Received 5 February 2013; published 1 August 2013)

\begin{abstract}
Thermionic electron guns are capable of operating at high average currents in a variety of vacuum electronic applications, including conventional microwave tubes, but have been replaced by laser photocathode injectors for most applications requiring high-brightness electron beams. However, while laser photocathode guns are capable of providing the very high-brightness beams, they provide an increased level of system complexity and do not extrapolate well to injectors for high average current applications requiring high beam quality. We are developing a $714 \mathrm{MHz}$ injector based on a gridded thermionic electron gun for these applications. This paper presents an experimental study, computer simulations, and analysis of the performance of an existing gridded thermionic electron gun as an injector prototype, and a design concept for an improved injector configuration based on these results.
\end{abstract}

DOI: 10.1103/PhysRevSTAB.16.083401

PACS numbers: 07.77.Ka, 29.27.Ac

\section{INTRODUCTION}

Radio-frequency linear accelerators are used to accelerate short bunches (microbunches) of electrons to high energy using the rf fields excited in standing-wave or traveling-wave structures. The first stage of an electron linear accelerator is an electron injector, beginning with a cathode that is the source of the electrons. The quality, bunch length, and timing of the electrons injected into the first rf cell are critical to determining the properties of the final high energy electron bunches that are generated by a complete accelerator system [1]. For applications that require high electron beam quality, the final beam quality requirements in the interaction region can be traced back to requirements on the electron microbunches injected into the first cell of the rf accelerating system [2].

Electron injectors can use several different types of cathodes to generate the electrons that are accelerated in the first rf cavity $[3,4]$. One approach is to use a thermionic cathode located on the axis of the upstream wall of the first accelerating cell. This approach is capable of producing high average currents, since such cathodes can operate at high current density (up to $\sim 10 \mathrm{~A} \mathrm{~cm}^{-2}$ ) while filling every rf bucket of the linac. However, injectors using a simple thermionic cathode exposed to the rf fields of the first accelerating cavity do not provide a means to gate the electron emission. As a result, electrons will be emitted whenever the rf phase accelerates electrons from the cathode surface. One result is that there is a large energy spread in the electrons that exit the first rf cell, as well as a large

Published by the American Physical Society under the terms of the Creative Commons Attribution 3.0 License. Further distribution of this work must maintain attribution to the author(s) and the published article's title, journal citation, and DOI. spread in the rf phase angle at which electrons enter subsequent $\mathrm{rf}$ cells. In addition, there is a large population of electrons that are accelerated from the cathode, but do not exit the cell before the oscillating rf fields push the electrons back to the cathode or to the cavity wall. In an injector operating at high average current, this backbombardment can damage the cathode and can constitute a significant thermal load on the cavity wall. For these reasons, it is desirable to gate the electron emission from the cathode into the first rf cavity.

Direct gating of a thermionic cathode has been used in some low average current applications by carrying out the gating using a high voltage pulse applied to a gridded cathode as part of an injector that operates at a subharmonic of the linac frequency, and then typically employing subharmonic bunching to increase the peak current injected into the linac [5-8]. Also, direct 1-GHz rf modulation of the grid has been employed [9], again followed by subharmonic bunching. However, operating the injector at the $n$th subharmonic of the linac frequency means that only every $n$th bucket of the linac will be filled, thus reducing the average current that the linac can deliver. Also, other means have been employed that involve slicing a short charge bunch from a longer electron pulse, and simply discarding the remainder of the electrons [10,11]. This latter approach is also not suitable for high average current injector applications.

The most common current approach to gating is to generate electron bunches using emission from photocathodes that are excited by laser radiation, with the electron emission controlled directly by the temporal structure of the laser pulse. Photocathodes can operate at high instantaneous currents and provide electron bunches that have both high quality and high charge per microbunch. For these reasons, they are generally the preferred approach for 
high-brightness applications $[3,4,12-16]$. However, the use of laser photocathodes is a complicated and expensive approach that does not easily scale to robust systems to produce high average beam currents, since there are limitations of the drive lasers as well as limitations due to the thermal loading and lifetime of the photocathodes. Some work has also taken place to partially gate the emission from thermionic cathodes by using a dual-frequency cavity with appropriate phasing of the two modes [17,18], to photogate the emission from thermionic cathodes by adding laser irradiation [19,20], and to make use of ballistic bunch compression of the bunches produced by ungated thermionic cathodes [21]. References [22,23] compare self-amplified spontaneous emission in an undulator from injectors either using a photocathode electron gun or using a thermionic rf gun combined with alpha magnet compression, and Ref. [24] presents a comparison of thermionic, photogated thermionic, and photocathode injectors in the context of $\mathrm{THz}$ radiation generation. Also, a dualfrequency cavity combined with a field-emitter array (FEA) has been proposed, with the combination of two harmonically related frequencies and appropriate amplitude and phase relationships combining with the nonlinear emission characteristics of the FEA to permit control of the emission phase [25].

As an alternative to these various methods of gating the emission, we are exploring an approach that uses a gridded thermionic electron gun that combines a high current, robust thermionic emitter with direct rf modulation of the grid at the linac frequency and higher harmonics in order to provide a high current injector that provides the gating required for injection into the proper rf phase of the first rf accelerating cavity. In this paper, we evaluate the potential of a gridded thermionic electron gun as the first stage of an accelerator injector system, as proposed in Ref. [2].

The plan of our program is to carry out experimental measurements and theoretical investigations on a commercially available gridded thermionic electron gun driven at $\sim 700 \mathrm{MHz}$, and to study electron bunch length, bunch charge, and transverse beam emittance as a function of the negative bias between the grid and cathode $\left(V_{\text {grid }}\right)$ and the rf drive power at gun voltages of 30-35 kV. Also, we want to test the effects of adding third harmonic drive to the cathode-grid circuit to reduce the electron bunch length. These measurements are then compared to simulations using the electron gun code MICHELLE [26] in order to model the diagnostics and to benchmark the simulations. MICHELLE simulations are also used to study an alternative electron gun design that can have improved transverse emittance and shorter electron bunch length.

The electron injector for a high average power rf linac should produce high charge electron bunches with low energy spread ready for injection to the linac. Typical beam parameters for such an injector are a bunch charge of $\sim 1 \mathrm{nC}$ at a repetition rate of $\sim 700 \mathrm{MHz}$ in cw operation.
Other proposed target parameters are a normalized transverse rms emittance of $\varepsilon_{\perp}<15 \mathrm{~mm}$ mrad and a rms bunch duration of $\tau_{b}<50 \mathrm{ps}$.

\section{EXPERIMENTAL SETUP}

The goal of the experiment is to characterize the performance of a gridded thermionic electron gun that is similar to the electron gun used in the production of inductive output tube amplifiers (IOTs) [27]. It incorporates a dispenser cathode and a pyrolytic graphite grid that can modulate the cathode emission at frequencies greater than $1 \mathrm{GHz}$. In an IOT, the modulated electron beam would then interact in a resonant output cavity in order to generate tens of kilowatts of rf power at the drive frequency at up to $100 \%$ duty factor. Here, the gun is being used as the prototype of an advanced thermionic gun that will be optimized for minimum bunch length and minimum normalized emittance while operating at $\sim 1 \mathrm{nC}$ microbunch charge in fully cw operation at $\sim 700 \mathrm{MHz}$.

A schematic cross sectional diagram of the electron gun is shown in Fig. 1. The gun incorporates a 3-cm diameter cathode and a closely spaced pyrolytic graphite grid that is biased negatively with respect to the cathode. The center of the cathode is $\sim 5 \mathrm{~cm}$ from the end of the gun output flange. A central hole $\sim 7 \mathrm{~mm}$ in diameter passes through the cathode and the grid wires, and is intended to reduce cathode damage due to ion back-bombardment. As part of an IOT, the electron gun would normally operate with negative DC high voltage on the cathode with respect to the anode, which is at ground potential, and with the emission controlled by the DC bias of the grid with respect to the cathode and by the rf drive of the cathode-grid circuit. However in our experiment, the cathode-grid circuit is pulsed to a negative high voltage ( $V_{\text {cathode }}$ ) with respect to the grounded anode of up to $-36 \mathrm{kV}$ using a high voltage modulator. The cathode heater is powered by a $60 \mathrm{~Hz}$ AC feed that floats on the high voltage pulse, and an adjustable negative grid bias $\left(V_{\text {grid }}\right)$ of up to $-180 \mathrm{~V}$

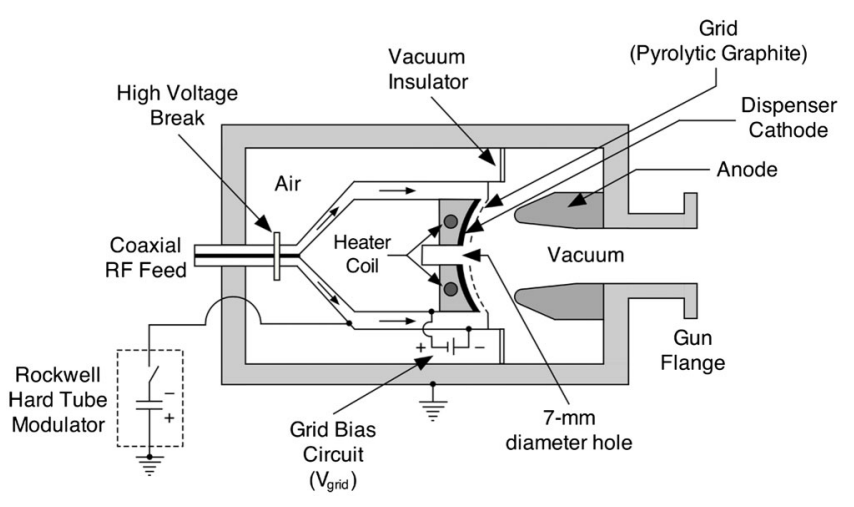

FIG. 1. Schematic diagram of the gridded thermionic electron gun. 


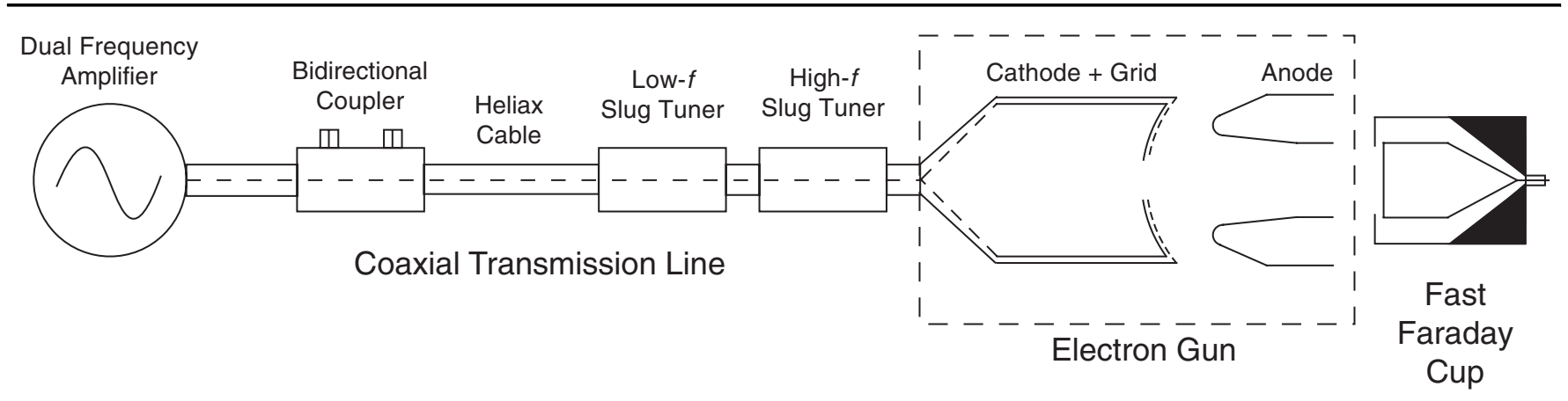

FIG. 2. Schematic diagram of the experimental setup for bunch length measurements.

with respect to the cathode also floats with the high voltage pulse. The negative bias on the grid, which suppresses electron emission, is combined with direct rf modulation of the cathode-grid circuit via a coaxial feed line to produce a high current electron beam that is bunched at the $\mathrm{rf}$ frequency. The $\mathrm{rf}$ modulation is introduced through a coaxial line that feeds the cathode-grid circuit through a high voltage coaxial break. Beyond the break, the coax opens up to enclose the cathode and then terminates at the grid, with the cathode-grid gap capacitively loading the end of the coaxial line. The goal of our experiments is to characterize the properties of the electron beam, first by depositing the electrons in a Faraday cup placed close to the output flange, in order to measure the temporal width and charge of electron bunches, and second by using slitbased optical measurements to determine the electron beam emittance.

The high voltage power supply for the IOT gun is a $70 \mathrm{kV}$ Rockwell hard tube modulator with an output pulse width that is variable up to $\sim 15 \mu \mathrm{s}$. For these experiments, the voltage was limited to $V_{\text {cathode }} \sim 36 \mathrm{kV}$ and the output pulse width was set at its maximum. The heater coil inside the cathode assembly is not compensated by a center return. As a result, it produces stray magnetic fields at the cathode surface that oscillate at $60 \mathrm{~Hz}$, producing a variation in the beam from the gun. In order to avoid this effect, the modulator timing was synchronized with the zero crossing of the heater current measured at the cathode. Experimental measurements were typically performed late in the pulse, in order to minimize the effect of gun turn-on transients and voltage ringing. These transients occur because the high voltage feed to the cathode is essentially a high voltage lead, rather than a transmission line, and is not matched to the cathode circuit. (Actually, there are three separate high voltage leads connected to the gun, all sharing an identical high voltage pulse from the modulator, but carrying separate interlead voltages to power the $\mathrm{AC}$ heater circuit and to created a DC bias of the grid with respect to the cathode bias, both of which float at the cathode potential.) In the case of rf drive, a 1- $\mu$ s rf pulse was applied to the grid circuit using the same timing near the end of the high voltage pulse, and fast time scale measurements were made in a window within the rf pulse.
Figure 2 shows the experimental setup used to measure the length and charge of the electron bunches from the gun. The rf drive for the cathode-grid circuit was produced by a Technical Services Laboratory Model 1710 dualfrequency amplifier that provides up to $\sim 500 \mathrm{~W}$ of output at a fundamental frequency tunable between 700 and $714 \mathrm{MHz}$ as well as up to $\sim 450 \mathrm{~W}$ at the third harmonic of the fundamental frequency. Both output signals are locked to a single $100 \mathrm{kHz}$ crystal oscillator, with separate controls of the two output power levels. A phase adjuster in the low frequency line allows control of the relative phase between the fundamental and third harmonic signals. Finally, the two signals are combined inside the chassis to feed a single coaxial output. The output power levels are read out directly from the amplifier using an internal peak power meter, and the rf is also monitored using a bidirectional coupler that is read out directly on a fast digital oscilloscope. The cathode-grid circuit is fed by a $50 \Omega$ Heliax cable connected to the coaxial output of the amplifier. The coaxial feed from the amplifier employs separate low and high frequency slug tuners in a series configuration for impedance matching both signals into the electron gun. For reasons of convenience, we chose to carry out these experiments at 714 and $2142 \mathrm{MHz}$.

The electron bunches from the gun were collected on the copper cone of a fast Faraday cup (Princeton Scientific Corp.) with $\sim 50 \mathrm{ps}$ time resolution. The Faraday cup includes a grid that is biased negative with respect to the copper cone in order to capture secondary electrons generated by the impact of beam electrons on the copper cone. The Faraday cup was placed as close as possible $(\sim 3 \mathrm{~cm})$ to the gun output flange in order to limit bunch spreading due to velocity spread and space-charge effects. The current collected by the Faraday cup passed through calibrated attenuators and was recorded by a Tektronix DSA 71604B 16-GHz digital oscilloscope, with the signal line terminated in $50 \Omega$. The Faraday cup remains near ground potential, and is separated from ground only by the voltage drop through the $50 \Omega$ termination. The experimental variables were the gun voltage, the grid bias voltage $V_{\text {grid }}$, and the level of fundamental and third harmonic rf drive. In addition, the heater power was controlled to ensure reproducible emission from the cathode. 


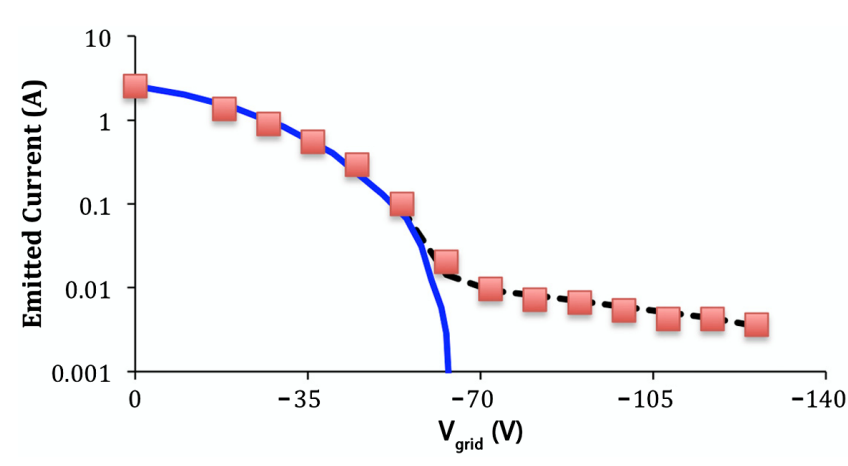

FIG. 3. Measured beam current versus $V_{\text {grid }}$ for $V_{\text {cathode }}=$ $-31 \mathrm{kV}$. The solid line is from a MICHELLE simulation of the electron gun, with emission only from the face of the cathode. The simulation model takes into consideration temperaturelimited emission from the cathode at $1095 \mathrm{~K}$. The dashed line includes emission from both the face of the cathode and the edge surface of the central hole through the cathode.

\section{DISCUSSION OF THE FARADAY CUP DATA}

Figure 3 shows the measured current from the Faraday cup as a function of $V_{\text {grid }}$ with $V_{\text {cathode }}=-31 \mathrm{kV}$. The peak current at $V_{\text {grid }}=0 \mathrm{~V}$ is $\sim 2.5 \mathrm{~A}$, which according to our simulations corresponds to mixed space-charge and temperature-limited emission from the cathode. However, as $V_{\text {grid }}$ is made increasingly negative, the emission from the cathode becomes space-charge limited, and falls rapidly until about $V_{\text {grid }}=-70 \mathrm{~V}$. Simulations show that emission from the face of the cathode through the grid should cut off sharply at this value of grid bias. However, the experimental data shows that the emission does not cut off at this value of $V_{\text {grid }}$. Instead, it continues to fall steadily, but at a slower rate than at lower values of grid bias, and there is still $3.6 \mathrm{~mA}$ of current at $V_{\text {grid }}=-126 \mathrm{~V}$. This behavior indicates that there is a source of unwanted emission in the gun that is not effectively controlled by the grid bias.

One possible source of the unwanted emission is emission from the grid wires, which can become contaminated with barium from the hot cathode and may be hot enough to emit. However, this explanation is not well supported by the data, which shows that the emission continues to fall monotonically as the grid bias is increased above the predicted cutoff value. Emission from the grid wires should not be affected in this way by the grid bias, since the change in voltage between the grid and anode is less than $1 \%$.

The second possibility is emission from the edge surface of the central hole through the cathode, which can readily be contaminated with barium, and which is clearly hot enough to emit, being at the same temperature as the rest of the cathode. Electron trajectories originating in this region and leaking through the central hole in the grid wires would still be affected by the grid potential, though to a lesser extent than emission from the face of the cathode, and should decrease monotonically as $V_{\text {grid }}$ is made more negative. This mechanism explains the data in Fig. 3, as shown in the dashed curve that is derived from a simulation that includes emission from the edge surface of the central hole through the cathode, and it also sheds light on the results of rf-gated Faraday cup measurements that are shown in later figures. In addition, further evidence of this phenomenon based on optical diagnostics will be presented later in this paper.

The phenomenon of current escaping through the central hole in the grid wires was discussed by Wright and coworkers as the source of interbunch emission in the presence of $\mathrm{rf}$ grid drive, based on their MICHELLE simulations of a similar IOT electron gun [27]. In the context of an IOT amplifier, this phenomenon will result in some electrons entering the output cavity in the accelerating phase of the cavity fields, and gaining energy as they transit the output cavity, resulting in increased $\mathrm{x}$-ray production when they are deposited in the IOT collector. However, Ref. [28] analyzed interbunch current only in the presence of $\mathrm{rf}$ drive. Our results, as well as the corresponding MICHELLE simulations, in which the edge of the cathode hole is permitted to emit, show that there is also a DC component of current emerging from the center hole with no rf drive and in the presence of strong negative grid bias.

In the IOT application, the gridded electron gun is typically used to create $\sim 180$ degree modulation of the electron beam, in order to efficiently generate high power rf in the output cavity [29]. However, the injector application requires the generation of much shorter electron bunches for injection into the optimum phase of a set of rf accelerating cavities. In the presence of a negative value of $V_{\text {grid }}$ combined with a single-frequency $r f$ drive, the cathode will emit electrons whenever the instantaneous value of the grid voltage is positive with respect to the cathode. As a result, the length of the electron bunches can be controlled by the ratio of the peak rf amplitude applied to the grid and the value of $V_{\text {grid }}$. This simple picture ignores transit-time effects in the cathode-grid gap. Nevertheless, as that ratio is decreased towards one, the bunch lengths are reduced, the peak bunch current is reduced, and for both reasons, the charge per bunch is also reduced.

One means to reduce the bunch length without simultaneously reducing the peak bunch current is to change the rf modulation waveform from sinusoidal to a more peaked waveform. One practical way to accomplish this is to add higher harmonic modulation to the grid drive. We chose to investigate third harmonic modulation, in this case at $2142 \mathrm{MHz}$, that is locked in phase with the fundamental harmonic modulation at $714 \mathrm{MHz}$. With the correct relative phase between the two signals, the rf modulation will become more peaked, and thus shorter bunches will in principle be produced at the same peak rf amplitude on the grid. In order to carry out this procedure, a special 


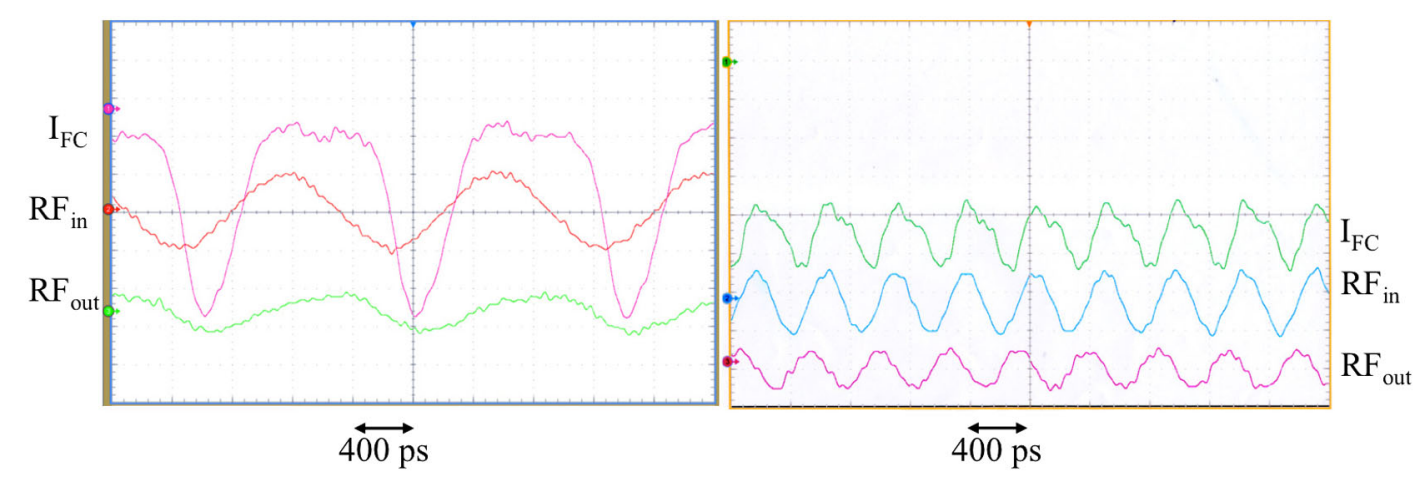

FIG. 4. Measured Faraday cup current $\left(I_{\mathrm{FC}}\right)$ for single-frequency $714 \mathrm{MHz}$ (left) and $2142 \mathrm{MHz}$ (right) grid drive. For the left-hand data set, the parameters are $V_{\text {cathode }}=-24 \mathrm{kV}, V_{\text {grid }}=-90 \mathrm{~V}$, and $\sim 100 \mathrm{~W}$ at $714 \mathrm{MHz}$ producing a $408 \mathrm{ps} \mathrm{FWHM}(\sim 170 \mathrm{ps}$ rms) micropulse with peak current $\sim 2.2 \mathrm{~A}$ and $0.9 \mathrm{nC}$ per bunch. For the right-hand data set, the parameters are $V_{\text {cathode }}=-24 \mathrm{kV}$, $V_{\text {grid }}=-63 \mathrm{~V}$, and $\sim 300 \mathrm{~W}$ at $2142 \mathrm{MHz}$, producing a peak current of $\sim 110 \mathrm{~mA}$. The colored dot on the left axis associated with each waveform shows the associated baseline of the trace. Also shown are the incident and reflected $\mathrm{rf}_{\mathrm{f}}$ waveforms, $\mathrm{rf}_{\text {in }}$ and $\mathrm{rf}_{\text {out }}$.

dual-frequency amplifier was purchased for this experiment, as described earlier in this paper.

One experimental question that needed to be addressed was to establish that the $2142 \mathrm{MHz}$ signal at the input to the electron gun would cause effective rf modulation of the voltage across the cathode-grid gap. Figure 4 addresses this issue. It shows the measured Faraday cup waveforms corresponding to single-frequency modulation at 714 and $2142 \mathrm{MHz}$. The $714 \mathrm{MHz}$ rf modulation of the grid produces strong modulation of the current measured by the Faraday cup, with a peak current of $\sim 2.2 \mathrm{~A}$ and $0.9 \mathrm{nC}$ per bunch. However, there is also evidence of interbunch current, as indicated by the downward shift of the signal from the baseline of the trace, which is indicated by the pink dot on the left axis. This current is believed to be related to the unmodulated current emitted from the central cathode hole, as discussed with reference to Fig. 3. The $2142 \mathrm{MHz}$ modulation also produces a modulation of the current measured by the Faraday cup, thus demonstrating that the $2142 \mathrm{MHz}$ modulation is appearing across the cathode-grid gap. However in this case the DC component, or interbunch current, is much larger than the modulated current. In this case, the baseline of the trace is indicated by the green dot on the left axis. This less effective bunching may be due to transit-time effects in the gap between the cathode and grid, which are more significant at the higher frequency. Also, the peak microbunch current of $110 \mathrm{~mA}$ is substantially lower than the 2.2 A current measured at $714 \mathrm{MHz}$, even though the $V_{\text {grid }}$ is less negative and the rf drive is substantially higher. In addition to possible transit-time effects, this appears to be due to the less effective coupling of rf power into the cathode-grid gap.

In order to estimate the effective coupling of an rf signal into the cathode-grid gap, we used a simple model of the rf input circuit as a $50 \Omega$ coaxial transmission line driving a purely capacitive load. (There will also be a resistive component when an electron beam is generated.) The cathode-grid region was modeled as two parallel $3-\mathrm{cm}$ disks separated by $250 \mu \mathrm{m}$, resulting in an estimated capacitance of $C=25 \mathrm{pF}$. The impedance of the capacitor is then $(i \omega C)^{-1}$, where $\omega=2 \pi f$, and $f$ is either 714 or $2142 \mathrm{MHz}$. In this case, for $100 \mathrm{~W}$ of rf drive, the cathodegrid voltage would be approximately $51 \mathrm{~V}$ at $714 \mathrm{MHz}$ and $8.5 \mathrm{~V}$ at $2142 \mathrm{MHz}$. These calculations do not include the effect of the slug tuners in the transmission line, which are always adjusted to minimize the reflected signal from the electron gun at both frequencies. However, they do suggest that the effective coupling to the cathode-grid gap will be substantially lower for the third harmonic signal.

The next test was to employ combined modulation of the cathode-grid circuit at both 714 and $2142 \mathrm{MHz}$. To optimize the combined waveforms, the relative phase of the two single-frequency components was adjusted to produce the shortest effective electron bunch lengths. Figure 5

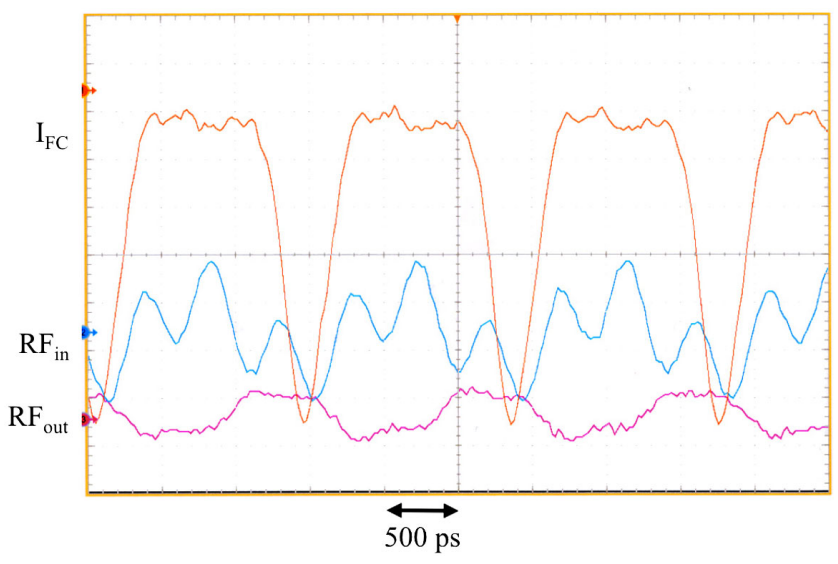

FIG. 5. Measured Faraday cup current, $I_{\mathrm{FC}}$, for combined first and third harmonic modulation of the electron gun. The parameters are $V_{\text {cathode }}=-36 \mathrm{kV}, V_{\text {grid }}=-90 \mathrm{~V}$, with $\sim 540 \mathrm{~W}$ at $714 \mathrm{MHz}$ and $\sim 450 \mathrm{~W}$ at $2142 \mathrm{MHz}$. The microbunches are 325 ps FWHM (135 ps rms), with 2.81 A peak current and $\sim 0.91 \mathrm{nC}$ per bunch. Also shown are $\mathrm{rf}_{\text {in }}$ and $\mathrm{rf}_{\text {out }}$. 

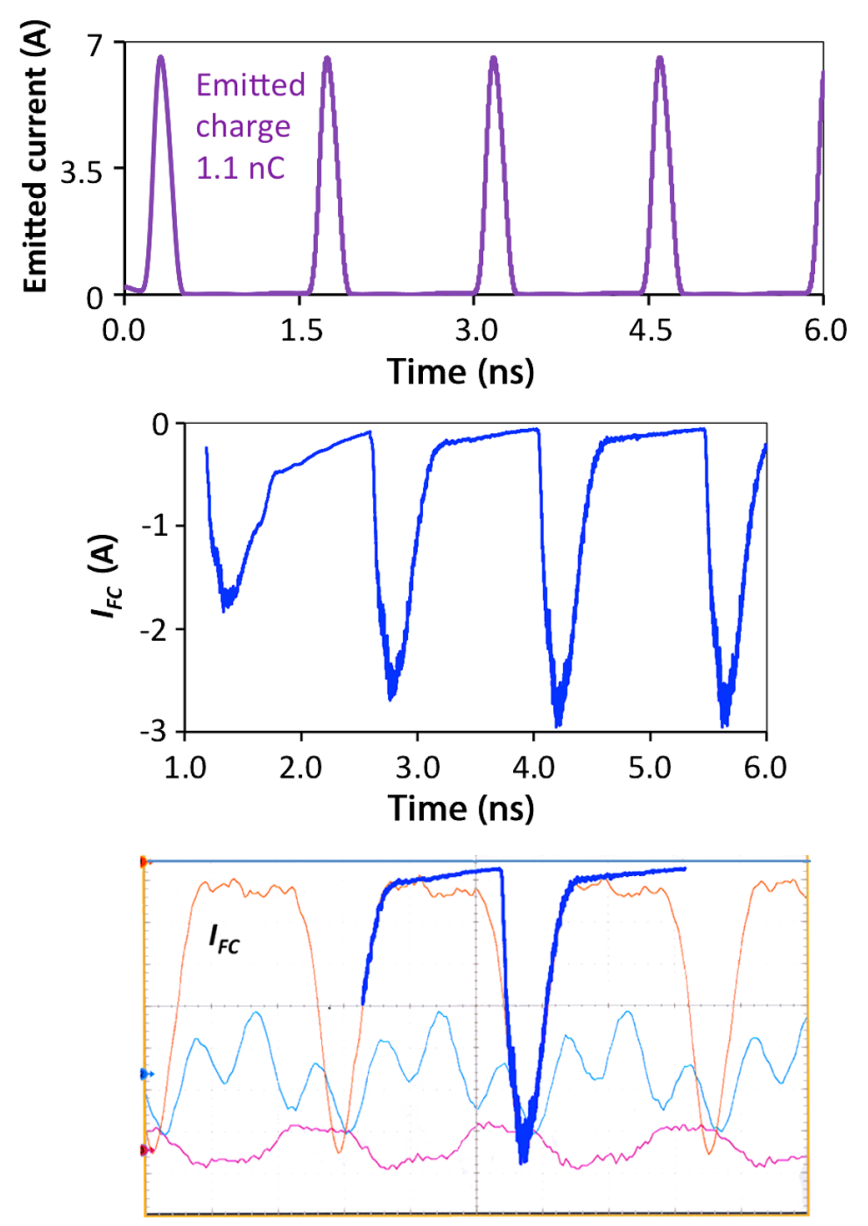

Time

FIG. 6. MICHELLE simulation of the bunched beam for operation corresponding to Fig. 5. (top) Emitted current from the cathode; (center) current reaching the Faraday cup; (bottom) comparison of simulation and experimental data from Fig. 5.

shows the Faraday cup measurements for $V_{\text {cathode }}=$ $-36 \mathrm{kV}, V_{\text {grid }}=-90 \mathrm{~V}$, with $\sim 540 \mathrm{~W}$ at $714 \mathrm{MHz}$ and $\sim 450 \mathrm{~W}$ at $2142 \mathrm{MHz}$. The microbunches are $325 \mathrm{ps}$ FWHM ( $\sim 135$ ps rms), with 2.81 A peak current and $\sim 0.91 \mathrm{nC}$ per bunch. Note that rms bunch durations are calculated assuming a Gaussian waveform with the

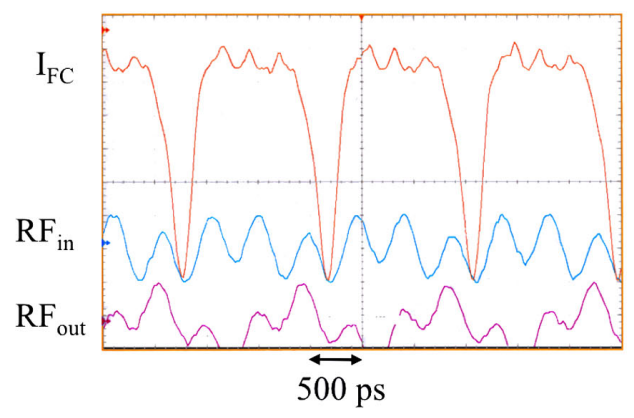

measured value of FWHM. As noted for the previous data, a small level of DC, or interbunch current, persists.

Figure 6 shows a MICHELLE simulation of the operation of the electron gun for parameters corresponding to Fig. 5, including first and third harmonic rf drive. However, since there is no direct experimental determination of the $\mathrm{rf}$ voltages in the cathode-grid gap, these voltages were set to $U_{1}=69 \mathrm{~V}$ and $U_{3}=23 \mathrm{~V}$ to provide a good fit to the data. Figure 6 (top) shows the emission from the cathode, which peaks at more than $6 \mathrm{~A}$, with $1.1 \mathrm{nC}$ charge per bunch. The FWHM of the cathode current bunch is $160 \mathrm{ps,}$ and the rms width is 74.7 ps. Figure 6 (center) shows the bunch shape at the position of the Faraday cup $(8 \mathrm{~cm}$ from the center of the cathode). Here, the peak bunch current is $2.96 \mathrm{~A}$, with $0.95 \mathrm{nC}$ charge per bunch. Also, the FWHM of the bunch is $301 \mathrm{ps}$. The rms bunch width is $160 \mathrm{ps}$, but is strongly influenced by the tail of the bunch, which corresponds to interbunch current. Note that the first two bunches are not typical, and the bunch is still evolving to the shape shown in the third and subsequent bunches. Figure 6 (bottom) shows a comparison of the third bunch to the bunches measured in Fig. 5.

One means to shorten the length of the electron bunches is to employ higher negative values of $V_{\text {grid }}$. Figure 7 shows data for $V_{\text {grid }}=-100 \mathrm{~V}$ (left) and $V_{\text {grid }}=-125 \mathrm{~V}$ (right). In order to optimize the bunches, the applied power at $714 \mathrm{MHz}$ was reduced to $\sim 200 \mathrm{~W}$, while the same $\sim 450 \mathrm{~W}$ was applied at $2142 \mathrm{MHz}$. For $V_{\text {grid }}=-100 \mathrm{~V}$, the electron bunches are $235 \mathrm{ps}$ FWHM ( $~ 98$ ps rms) and the peak bunch current is $580 \mathrm{~mA}$, corresponding to $\sim 135 \mathrm{pC}$ per bunch. For $V_{\text {grid }}=-125 \mathrm{~V}$, the bunch length is reduced to 190 ps FWHM (80 ps rms), but the peak bunch current drops to $74 \mathrm{~mA}$, corresponding to only $14 \mathrm{pC}$ per bunch. The rapid falloff of peak bunch current and charge per bunch at higher levels of negative grid bias shows the limitation of this approach when applied to the existing electron gun. However, these measurements also document the capability of the Faraday cup to measure these short bunches, indicating that the measured bunch shapes shown in Fig. 5 should not have been broadened by the diagnostics.

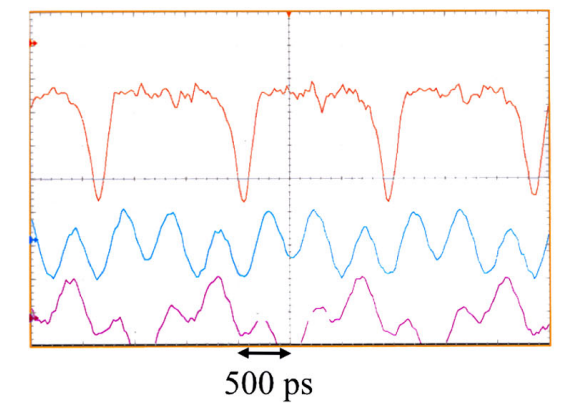

FIG. 7. Faraday cup data for combined first and third harmonic modulation of the electron gun and higher negative grid bias for $V_{\text {cathode }}=-36 \mathrm{kV}, \sim 200 \mathrm{~W}$ at $714 \mathrm{MHz}$ and $\sim 450 \mathrm{~W}$ at $2142 \mathrm{MHz}$. The left traces correspond to $V_{\text {grid }}=-100 \mathrm{~V}$; the right traces correspond to $V_{\text {grid }}=-125 \mathrm{~V}$. 


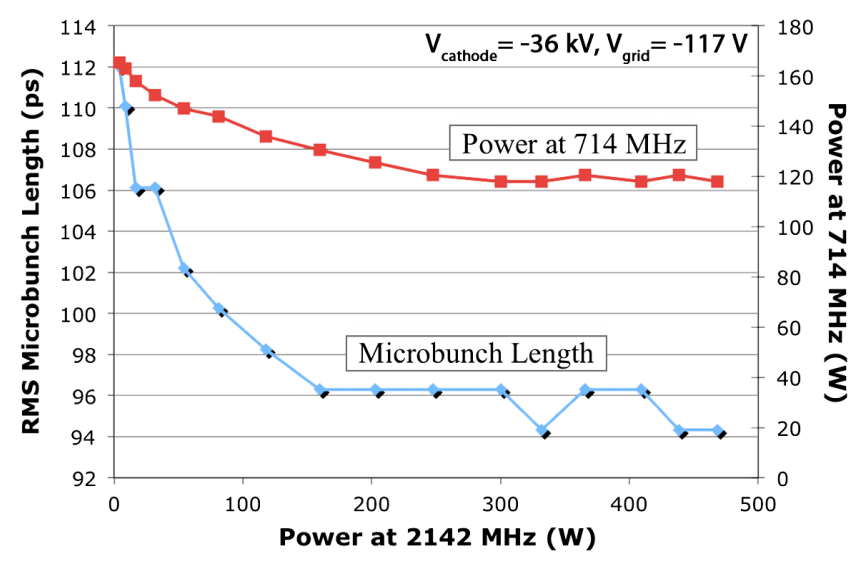

FIG. 8. The rms bunch length and first harmonic power versus third harmonic power at $0.6 \mathrm{~A}$ peak microbunch current.

In order to examine the effect of adding $2142 \mathrm{MHz}$ modulation to the grid, we took a set of data at $V_{\text {cathode }}=$ $-36 \mathrm{kV}$ and $V_{\text {grid }}=-117 \mathrm{~V}$ in which the peak microbunch current was set to $0.6 \mathrm{~A}$ with only $714 \mathrm{MHz}$ modulation applied, and then various levels of $2142 \mathrm{MHz}$ modulation were added, and the $714 \mathrm{MHz}$ modulation decreased, in order to maintain $0.6 \mathrm{~A}$ of peak microbunch current. The results are shown in Fig. 8. With no third harmonic drive, approximately $165 \mathrm{~W}$ of power at $714 \mathrm{MHz}$ was required, resulting in an rms bunch width of $\sim 112$ ps. The required first harmonic power drops monotonically as the third harmonic power is increased, and is reduced to $\sim 120 \mathrm{~W}$ with $\sim 200 \mathrm{~W}$ at third harmonic, resulting in a microbunch length of $\sim 96 \mathrm{ps}$ rms. Neither the first harmonic power required to produce $0.6 \mathrm{~A}$ peak bunch current at this value of negative grid bias nor the rms bunch length change much when the third harmonic drive is further increased in steps up to $\sim 475 \mathrm{~W}$. This data demonstrates that increasing the ratio of third to first harmonic power can help shorten the rms length of the

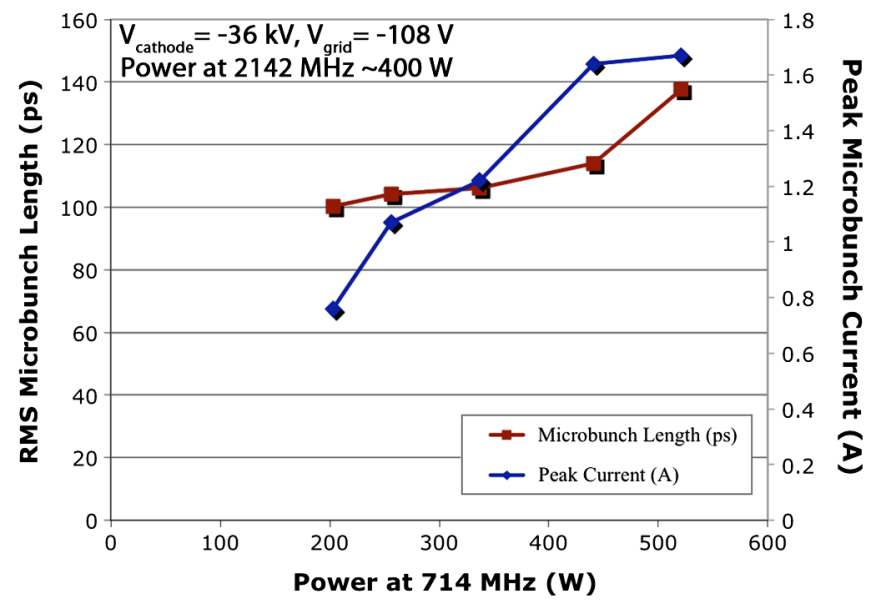

FIG. 9. Peak current and microbunch length versus power at $714 \mathrm{MHz}$. electron bunches for a fixed value of the peak microbunch current.

Figure 9 shows a plot of peak microbunch current and microbunch length versus the power applied at $714 \mathrm{MHz}$ with the $2142 \mathrm{MHz}$ power set at $\sim 400 \mathrm{~W}$ and the grid bias set to $V_{\text {grid }}=-108 \mathrm{~V}$. Both the bunch length and the peak microbunch current increase as the first harmonic drive power is increased.

\section{DISCUSSION OF THE EMITTANCE DATA}

Aside from bunch length and bunch charge, the remaining critical parameter for the bunches produced by the electron gun is their transverse emittance. For our low energy beam, we chose to employ a slit-base measurement [30-32], in which a $100 \mu \mathrm{m}$ horizontal tantalum slit was scanned across the beam using a vertical translator and the electrons passing through the slit were intercepted by a YAG:Ce fluorescent screen. The fluorescent screen was also mounted on a vertical translator, in order to keep it centered on the resulting electron trajectories. In turn, the fluorescent screen was imaged and recorded using a digital CCD camera (FOculus camera model FO531TB) controlled by LABVIEW ${ }^{\mathrm{TM}}$ software. The camera was triggered electronically. The exposure time was set to $\sim 3 \mu \mathrm{s}$, which permitted the camera to discriminate against emission that did not occur during the typical $1-\mu$ s rf drive pulse. The low beam energy and the beam divergence make it difficult to carry out accurate measurements, since the most divergent electron trajectories may intercept the wall of the vacuum vessel before reaching the slit plane, and some trajectories through the slit may also intercept the wall before reaching the YAG screen. Several different experimental setups were used, in an attempt to optimize the acceptance of divergent electron trajectories.

Figure 10 shows a schematic of the final experimental setup that was employed. In this setup, a solenoidal lens was added to help collimate the beam, thus ensuring that more of the beam reached the slit plane, and that more of the electrons that passed through the slit reached the YAG screen. The effective geometry from the gun up to the slit plane is shown in Fig. 11, which also shows a MICHELLE simulation of the electron trajectories from the gun, both without lens current (center), and with a lens current of $1.65 \mathrm{~A}$ (top). In these simulations, the gun is modeled for $V_{\text {cathode }}=-32 \mathrm{kV}$ and $V_{\text {grid }}=-90 \mathrm{~V}$ with only first harmonic rf drive applied. Since we cannot directly measure the grid modulation amplitude resulting from a particular rf power from the amplifier, the rf modulation of the cathodegrid gap was adjusted in the simulation to produce a peak microbunch charge of $\sim 0.75 \mathrm{nC}$. Figure 11 shows that even at $I_{\text {lens }}=1.65 \mathrm{~A}$, some electron trajectories are lost to the wall before reaching the slit plane. In examining Fig. 11, it is important to note that the trajectories do not carry equal amounts of charge. Figure 11 (bottom) shows a simulation of the trajectories with no lens current, but with 


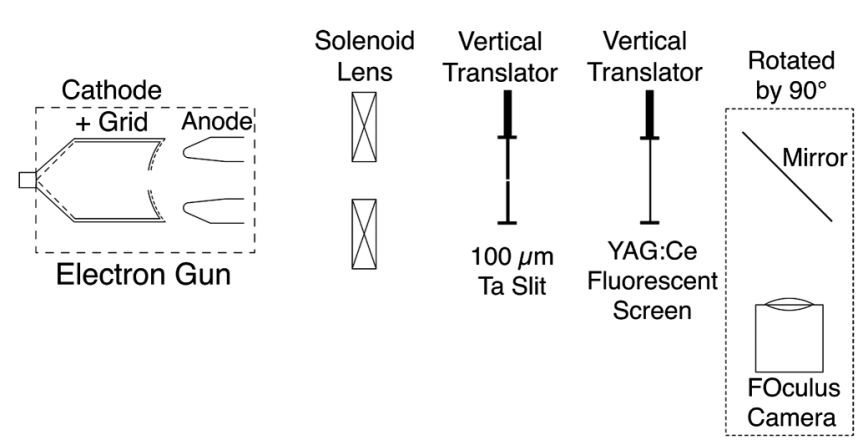

FIG. 10. Schematic of experimental setup for emittance measurements (not to scale). The vacuum enclosure is not shown.

the emission from the edge of the cathode hole suppressed. A comparison of the bottom and center simulations shows the affect of emission from the edge of the cathode hole in creating interbunch current.

Figure 12 shows simulations of the emittance measurements at $V_{\text {cathode }}=-32 \mathrm{kV}$ for $I_{\text {lens }}=1.65 \mathrm{~A}$ and $I_{\text {lens }}=$ $0 \mathrm{~A}$. The upper plot shows the normalized rms transverse emittance and the lower plot shows the corresponding bunch charge as a function of axial position. At location 1, electrons begin to hit the wall, and as a result the emittance of the bunch begins to drop. However, it is also important to note that the most divergent trajectories originate from the hole through the anode, and correspond to unmodulated emission from the gun, as shown in the bottom simulation shown in Fig. 11. In Sec. VI, we will discuss the design of a new electron gun configuration that eliminates this central hole, and therefore should eliminate the most divergent trajectories.

The lower plot in Fig. 12 shows that at $I_{\text {lens }}=1.65 \mathrm{~A}$, the solenoidal lens reduces the loss of bunch charge as a function of distance from the gun. This is most apparent downstream from the lens. However, the simulations also predict that the lens itself causes the bunch emittance to increase somewhat, as shown in the upper plot of Fig. 12.

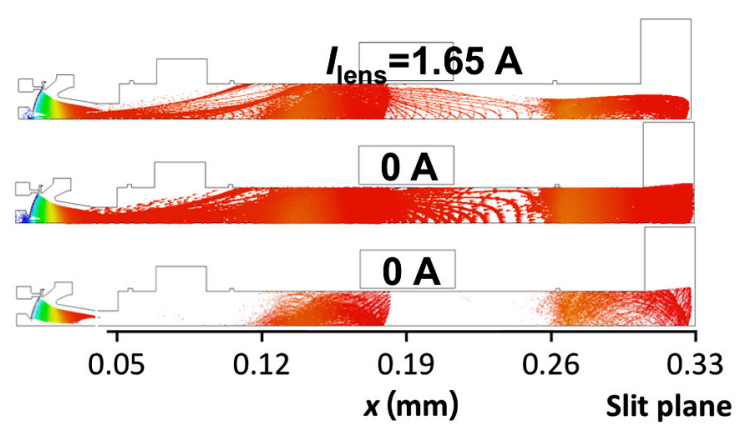

FIG. 11. Simulation of the emission measurements at $V_{\text {cathode }}=-32 \mathrm{kV}$ and effect of the emission from the central hole. Electron trajectories for: (top) $I_{\text {lens }}=1.65 \mathrm{~A}$ and (center) $I_{\text {lens }}=0 \mathrm{~A}$ include emission from the central hole; (bottom) $I_{\text {lens }}=0 \mathrm{~A}$ is with emission from the central hole suppressed.

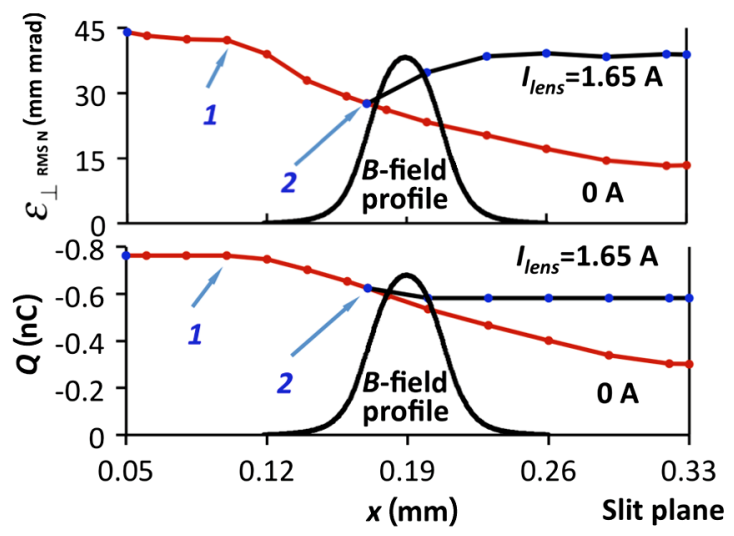

FIG. 12. Simulation of the emission measurements at $V_{\text {cathode }}=-32 \mathrm{kV}$. Normalized rms transverse emittance (top) and bunch charge (bottom) as a function of axial position for $I_{\text {lens }}=1.65 \mathrm{~A}$ and $I_{\text {lens }}=0 \mathrm{~A}$. Particles begin hitting the wall at the point labeled 1 and the magnetic field begins to affect the emittance at the point labeled 2 . The normalized magnetic field profile of the lens is also shown.

Figure 13 shows a comparison of the electron bunches at the exit of the gun, with and without emission from the central hole in the cathode. These simulations correspond to the center and bottom simulations in Fig. 11. The bunch charge with emission from the central hole is $0.77 \mathrm{nC}$ at a peak current of $2.06 \mathrm{~A}$, an rms bunch width of $166.5 \mathrm{ps}$, and a FWHM of 374 ps. Without emission from the central hole, the bunch charge is $0.74 \mathrm{nC}$, the peak current is $1.37 \mathrm{~A}$, the rms bunch width is $117 \mathrm{ps}$, and the FWHM is $465 \mathrm{ps}$, reflecting a more rectangular bunch shape. Note that the interbunch current vanishes completely before the start of the next bunch, once emission from the central hole is removed from the simulation.
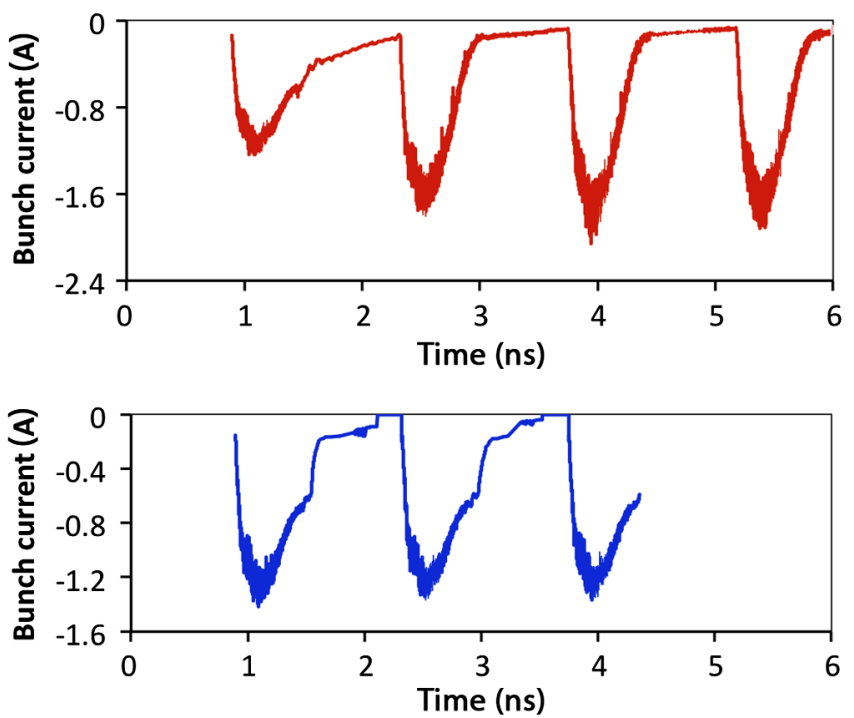

FIG. 13. Comparison of electron bunches at the gun exit with emission from the central hole (top) and without emission from the central hole (bottom). 


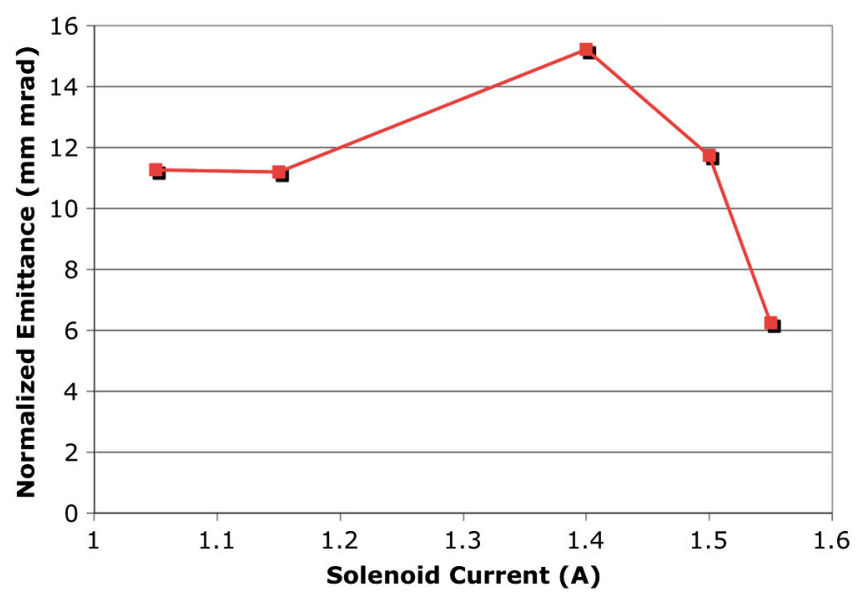

FIG. 14. Measured emittance versus solenoid current for $V_{\text {cathode }}=-32 \mathrm{kV}, V_{\text {grid }}=-90 \mathrm{~V}$, and $\sim 250 \mathrm{~W}$ of rf drive at $714 \mathrm{MHz}$.

Figure 14 shows a summary of the results of slit-based emittance measurements for $V_{\text {cathode }}=-32 \mathrm{kV}, V_{\text {grid }}=$ $-90 \mathrm{~V}$, and $\sim 250 \mathrm{~W}$ of rf drive at $714 \mathrm{MHz}$. Except for the values of $I_{\text {lens }}$, these experimental parameters correspond closely to the simulations shown in Figs. 11 and 12. The highest value of the measured normalized transverse rms emittance, as a function of magnet current, is $\sim 15 \mathrm{~mm}$ mrad, somewhat lower than predicted by the simulations. Also, the measured emittance drops off at higher values of the magnet current, while the simulations suggest that the emittance should increase. This may result from experimental factors that affect the measurement, including (1) the possibility that electron trajectories through the slit intercept the wall of the vacuum vessel before reaching the YAG screen and (2) effects due to the finite dynamic range of the optical measurements, which may result in a faint beam halo not being recorded by the measurement system.

An explanation of the DC current at high negative values of $V_{\text {grid }}$ that are reported from the Faraday cup measurements was provided by optical measurements of the beam that were recorded as part of the slit-based emittance measurements that included the use of a solenoidal lens to collimate the beam. With the slit on or near the beam axis, and $V_{\text {grid }}=-90 \mathrm{~V}$, the slit image was measured on the YAG screen at several different values of the magnet current while rf drive was present. In most cases, only the downstream image of the slit was recorded with the transverse spread of the slit image related to the beam emittance. However, at a small range of values of the magnet current, a small circle ( $\sim 4$-mm diameter) appeared on the YAG in addition to the slit image (see Fig. 15); when the rf drive was turned off, only the circle remained. The circle could be brought into sharp focus on the YAG at $1.15 \mathrm{~A}$ of solenoid current. This indicated that the source of the electron trajectories should correspond to a well-defined

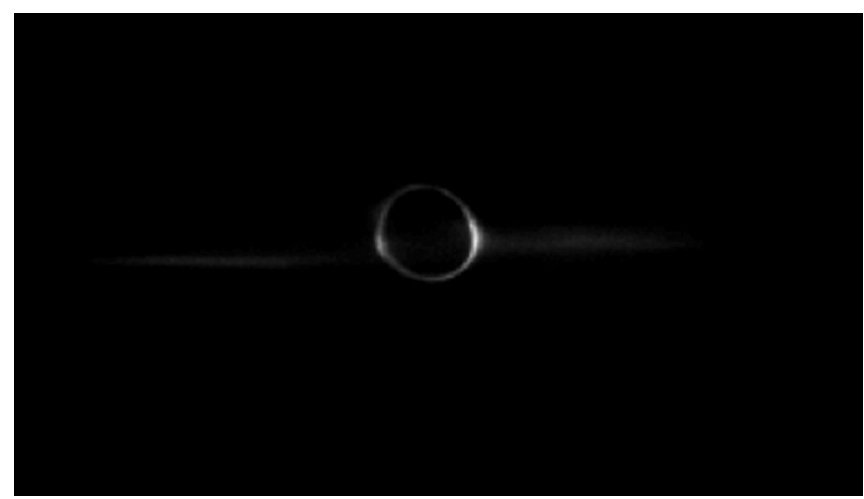

FIG. 15. Image of YAG plate at $I_{\text {lens }}=1.15$ A with a $100-\mu \mathrm{m}$ Ta slit on the beam axis, showing the circular beam pattern resulting from emission from the central hole in the cathode. The faint horizontal line is produced by the residual electrons emitted from the entire cathode surface and passing through the slit.

feature on the cathode whose emission was not strongly suppressed by the large negative values of $V_{\text {grid }}$. The only candidate feature is the edge of the central hole in the cathode. This region could emit electrons because of barium migration from the face of the cathode, and electron trajectories originating from this region could easily pass through the hole in the center of the grid pattern.

Since the focal length of the lens is known at the electron energy and magnet current employed, the electron energy is constant when in the field of the magnet, and the distance from the lens to the YAG is also known, one can estimate the distance to the apparent source of the electron trajectories imaged onto the circle assuming the thin lens approximation and using Eq. (1) [33]:

$$
f=\frac{4}{\int d z\left[q B_{z}(0, z) / \gamma m_{e} v_{z}\right]^{2}}
$$

Here, $B_{z}$ is the axial magnetic field of the focusing magnet as a function of position along the axis, $\gamma$ is the relativistic factor of the electrons, $m_{e}$ is the electron mass, and $v_{z}$ is the electron axial velocity. The integration is performed along the axis through the region where the magnetic field is nonzero. Using the focal length of the magnetic lens for $32 \mathrm{keV}$ electrons, it was determined that the apparent source of the electron trajectories was $\sim 30 \mathrm{~cm}$ behind the cathode, a physical location that clearly cannot emit electrons. The explanation for this is that the electron trajectories emitted from the inner edge of the cathode hole have a strong radial component, but are bent strongly in the vicinity of the cathode until they are close to paraxial straight lines as they exit the gun. The lens formula projects those lines back to their apparent source, which is well behind the plane of the cathode hole. 


\section{IMPROVED ELECTRON GUN WITH CATHODE INTEGRATED INTO THE ACCELERATING CAVITY}

The experimental measurements presented earlier in this paper demonstrate the capabilities of a gridded thermionic cathode as the first stage of an electron injector, but also showed that the existing electron gun, which was designed for an entirely different application, falls somewhat short of the typical emittance and bunch length parameters that are required for some rf linac application. The same MICHELLE/ANALYST [26,34] code combination that was used to model the existing gun was also used to design a new improved gun that could be capable of meeting those requirements. Some of the changes are straightforward, such as removing the central hole to eliminate the interbunch current, and reducing the cathode diameter in order to lower the emittance, which requires operating at higher mean emission current density. However, the next step was to envision an electron gun in which the initial DC acceleration between the grid plane and the anode, prior to injection into an rf cavity, is replaced by acceleration using the rf fields of the first cavity. Some preliminary work on this concept was reported in Ref. [35].

The basic concept of this novel rf-gated thermionic electron injector is shown in Fig. 16. It has a cylindrical rf accelerating cavity with the thermionic electron gun mounted in the first end wall on the cavity axis. Radiofrequency power is fed into the cavity through side power ports (not shown in the picture) and excites the $\mathrm{TM}_{010}$ resonant mode of the cavity. The $\boldsymbol{E}$ field of the mode has its maximum on the cavity axis and accelerates the electron bunches extracted from the cathode through the grid. The gun has a concave spherical cathode and rf modulated grid, with coaxial grid drive for $\mathrm{rf}$ excitation of the cathode-grid gap. A positive bias potential $U_{0}$ relative to the grid is
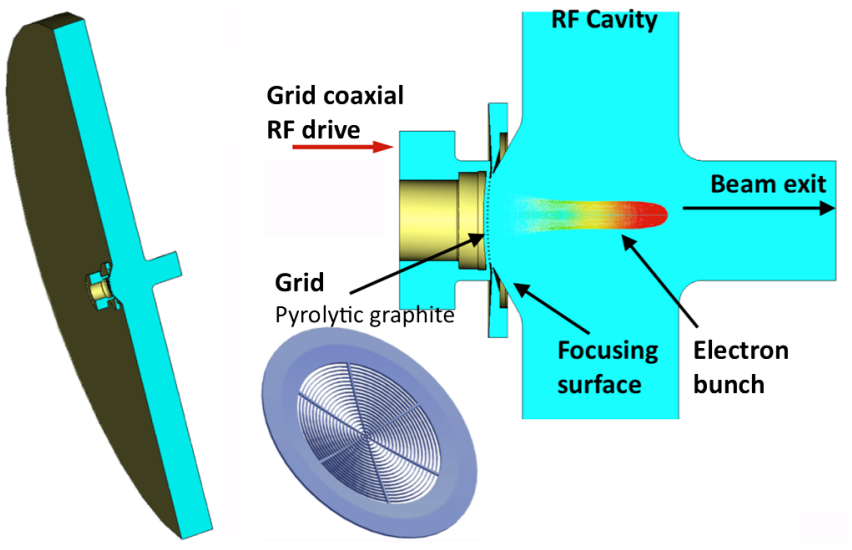

FIG. 16. (Left) Cut-away view of the schematic of a $700 \mathrm{MHz}$ rf-gated thermionic electron gun integrated into a cylindrical rf accelerating cavity. The power port is not shown. (Right) Closeup view of the central region of the cavity, showing the cathode and the holeless grid (inset), and the transit of a electron bunch. applied to the cathode and prevents emission if no $\mathrm{rf}$ excitation voltage $U_{1}$ is applied across the grid to cathode region. Radio-frequency excitation of the coaxial grid drive produces $\mathrm{rf}$ modulation of the voltage across the cathode beam extraction region. The rf excitation can be the first harmonic of the frequency $f$ of the main accelerating cavity $\mathrm{TM}_{010}$ resonant mode or can include higher harmonics of this frequency. To the right of the grid, cavity rf fields accelerate the electron bunches created by the cathode and grid.

The amplitude $U_{n}$ of the rf excitation is selected to provide the electric field for extraction of electron bunches of the required charge and duration. Higher positive bias voltage $U_{0}$ in general leads to shorter bunch duration and also requires higher harmonic rf voltage to keep the bunch charge unchanged. The grid to cathode spacing is selected to keep all the DC and $\mathrm{rf} \boldsymbol{E}$ fields below the voltage breakdown threshold, e.g. $70 \mathrm{kV} / \mathrm{cm}$. In addition the spacing determines the rf power needed to excite the gap. Our simulations show that the grid to cathode spacing has little effect on the emittances that are attainable. The size of the grid wires affects both the emittance of the electron beam and the structural strength of the grid. A grid with thinner wires produces smaller distortion of the $\boldsymbol{E}$ field and therefore allows smaller emittance. However, the grid wires must remain thick enough for structural integrity.

The key parameters responsible for the bunch properties and control are the DC bias voltage and different combinations of the harmonic amplitudes and phases. This includes the simplest case with only first harmonic for the grid rf drive excitation. Higher order harmonics fundamentally allow shorter electron bunches. However, shorter bunches require higher peak currents to achieve the same microbunch charge, and thus either a larger cathode diameter or higher peak current density from the cathode surface. In turn, a larger cathode diameter will tend to increase the transverse emittance, while operating at higher

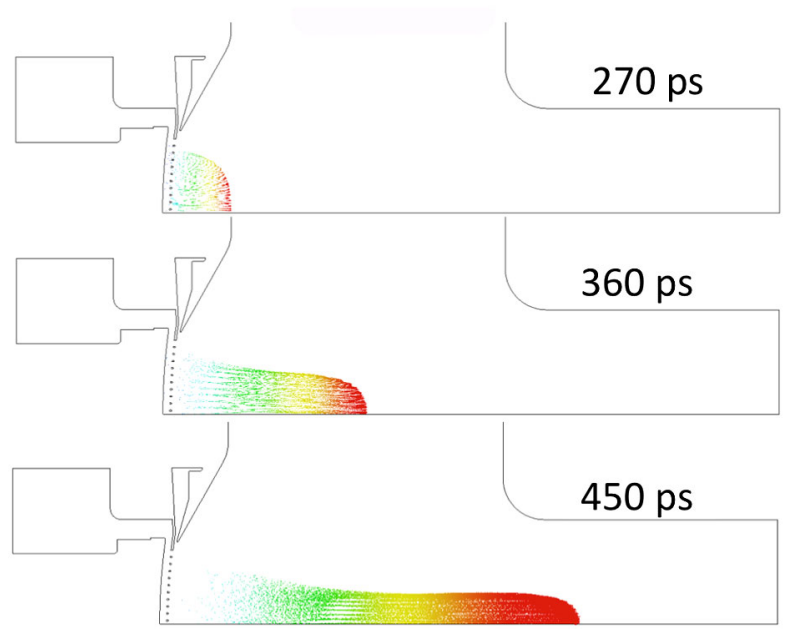

FIG. 17. Beginning stages of bunch formation. 
peak current densities would require operation at higher cathode temperatures, with a negative effect on cathode lifetime. An example of the operation of the new rf-gated bunched thermionic electron gun is shown in Fig. 17, where the beginning stages of the bunch formation are shown. An optimized geometry and optimized bunch extraction $\mathrm{rf}$ drive excitation conditions were used in the simulation $700 \mathrm{MHz}$, with combined first and third harmonic grid drive, to produce about $1 \mathrm{nC}$ of charge with an rms bunch width of $46 \mathrm{ps}$, normalized transverse and longitudinal emittances of $11.5 \mathrm{~mm}$ mrad and $40.5 \mathrm{kV} \mathrm{ps}$, and an energy of $120 \mathrm{keV}$.

\section{CONCLUSIONS}

In this paper, we have presented studies of the performance characteristics of a $35-\mathrm{kV}$ gridded thermionic electron gun in order to evaluate its potential as the prototype for an advanced thermionic electron gun for future accelerator injectors. The studies were carried out at $714 \mathrm{MHz}$, and were used to characterize the electron microbunch charge, microbunch length, and transverse emittance as a function of electron gun voltage, negative grid bias, and rf grid drive, including the effect of adding a third harmonic rf signal to the grid circuit. In studies using a fast Faraday cup, the gun has demonstrated microbunches with a microbunch length of 325 ps FWHM ( 135 ps rms), with $2.81 \mathrm{~A}$ peak current and $\sim 0.91 \mathrm{nC}$ per bunch. The use of combined first and third harmonic grid drive has been shown to reduce the length of the microbunches compared to pure first harmonic modulation. We also carried out slit-based measurements of the normalized transverse emittance of electron bunches from the gun, and measured typical values in the range of $10-15 \mathrm{~mm}$ mrad. However, these measurements were measured downstream from the gun, and may underestimate the transverse emittance at the gun exit plane due to beam scraping in transport. The emittance and bunch length measurements were compared to the predictions of detailed simulations using the electron gun code MICHELLE. Both the measurements and the simulations have demonstrated the potential of a gridded thermionic electron gun as the first stage of a high average current injector, but have also shown that the existing electron gun will need some modifications to further improve the beam parameters. We also presented an alternative injector configuration with a modified cathode geometry that makes use of direct $\mathrm{rf}$ acceleration of the beam from the gridded thermionic cathode. This alternative configuration offers the possibility of improved performance for the intended application.

\section{ACKNOWLEDGMENTS}

The authors are grateful for the assistance of L. Chen in developing an image correction software program for the
FOculus camera. This work was supported by the Office of Naval Research and the High Energy Laser Joint Technology Office. B. Zhou acknowledges the support of the Directed Energy Scholar internship program.

[1] T. Wangler, RF Linear Accelerators (Wiley, New York, 1998).

[2] P. Sprangle, J. Peñano, B. Hafizi, D. Gordon, S. Gold, A. Ting, and C. Mitchell, Phys. Rev. ST Accel. Beams 14, 020702 (2011).

[3] A. Todd, Nucl. Instrum. Methods Phys. Res., Sect. A 557, 36 (2006).

[4] I. Ben-Zvi and I. V. Bazarov, Nucl. Instrum. Methods Phys. Res., Sect. A 557, 337 (2006).

[5] A. Yeremian, J. Adamski, R. Kennedy, W. Gallagher, and J. Orthel, in Proceedings of the 1989 Particle Accelerator Conference (IEEE, Piscataway, NJ, 1989), pp. 657-659.

[6] J. M. J. Madey, G. J. Ramian, and T. I. Smith, IEEE Trans. Nucl. Sci. 27, 999 (1980).

[7] T. I. Smith, in Physics of Quantum Electronics, edited by S. F. Jacobs et al. (Addison-Wesley, Reading, MA, 1982), Vol. 8, pp. 77-87.

[8] B. A. Baklakov et al., Nucl. Instrum. Methods Phys. Res., Sect. A 470, 60 (2001); V. P. Bolotin et al., Nucl. Instrum. Methods Phys. Res., Sect. A 557, 23 (2006).

[9] R. J. Bakker, C. A. J. van der Geer, A.F. G. vanderMeer, P.W. van Amersfoort, W. A. Gillespie, and G. Saxon, Nucl. Instrum. Methods Phys. Res., Sect. A 307, 543 (1991).

[10] K. Togawa, T. Shintake, T. Inagaki, K. Onoe, T. Tanaka, H. Baba, and H. Matsumoto, Phys. Rev. ST Accel. Beams 10, 020703 (2007).

[11] T. Shintake et al., Phys. Rev. ST Accel. Beams 12, 070701 (2009).

[12] G. R. Neil et al., Phys. Rev. Lett. 84, 662 (2000).

[13] K. L. Jensen, N. A. Moody, D. W. Feldman, E. J. Montgomery, and P.G. O'Shea, J. Appl. Phys. 102, 074902 (2007).

[14] C. Hernandez-Garcia, T. Siggins, S. Benson, D. Bullard, H. F. Dylla, K. Jordan, C. Murray, G. R. Neil, M. Shinn, and R. Walker, in Proceedings of the 21st Particle Accelerator Conference, Knoxville, 2005 (IEEE, Piscataway, NJ, 2005), p. 3117; C. Hernandez-Garcia, P. G. O'Shea, and M.L. Stutzman, Phys. Today 61, No. 2, 44 (2008).

[15] J.G. Power, in Advanced Accelerator Concepts: 14th Advanced Accelerator Concepts Workshop, Annapolis, MD, 2010, AIP Conf. Proc. No. 1299, edited by S.H. Gold and G. S. Nusinovich (AIP, Melville, NY, 2010), pp. 20-28 [http://dx.doi.org/10.1063/1.3520316].

[16] B. E. Carlsten, Nucl. Instrum. Methods Phys. Res., Sect. A 285, 313 (1989).

[17] J. Edelen, S. Biedron, and S. Milton, in Proceedings of the Fifteenth Annual Directed Energy Symposium (Directed Energy Professional Society, Albuquerque, NM, 2012).

[18] J. Lewellen (unpublished); (private communication).

[19] Y.-E. Sun, J.W. Lewellen, and D.W. Feldman, in Proceedings of LINAC 2006 (ORNL, Knoxville, TN, 
2006), pp. 349-351 [http://accelconf.web.cern.ch/ AccelConf/106/PAPERS/TUP045.PDF].

[20] J. G. Neumann, J. R. Harris, B. Quinn, and P. G. O'Shea, Rev. Sci. Instrum. 76, 033303 (2005).

[21] J. W. Lewellen and S. Milton, Proc. SPIE Int. Soc. Opt. Eng. 3154, 162 (1997).

[22] S. V. Milton et al., in Proceedings of the European Particle Accelerator Conference, Vienna, 2000 (EPS, Geneva, 2000), pp. 755-757.

[23] N. D. Arnold et al., Nucl. Instrum. Methods Phys. Res., Sect. A 475, 28 (2001).

[24] S. G. Biedron, J. W. Lewellen, S. V. Milton, N. Gopalsami, J. F. Schneider, L. Skubal, Y. Li, M. Virgo, G. P. Gallerano, A. Doria, E. Giovenale, G. Messina, and I. P. Spassovsky, Proc. IEEE 95, 1666 (2007).

[25] J. W. Lewellen and J. Noonan, Phys. Rev. ST Accel. Beams 8, 033502 (2005).

[26] J. Petillo, E. Nelson, J. DeFord, N. Dionne, and B. Levush, IEEE Trans. Electron Devices 52, 742 (2005).
[27] http://www.cpii.com/product.cfm/1/28.

[28] E. Wright, K.T. Nguyen, J. A. Pasour, S. J. Cooke, B. Levush, J. J. Petillo, I. A. Chernyavskiy, J. B. DeFord, and B.L. Held, in Proceedings of the 2009 Particle Accelerator Conference, Vancouver, BC, Canada (IEEE, Piscataway, NJ, 2009), pp. 767-769.

[29] Y. Li, EUROFEL-Report-2007-DS5-074, 2007.

[30] M. Zhang, Fermi National Accelerator Laboratory Report No. FERMILAB-TM-1988, 1996.

[31] M. Reiser, Theory and Design of Charged Particle Beams (Wiley, New York, 2008).

[32] J. Safranek and P. M. Stefan, in Proceedings of the Fifth European Particle Accelerator Conference EPAC'96, Sitges, Spain, 1996, paper TUP006L.

[33] S. Humphries, Principles of Charged Particle Acceleration (John Wiley and Sons, New York, 1986).

[34] AWR Corp, Microwave J. 52, 104 (2009).

[35] C. Mitchell, P. Sprangle, and J. Peñano, IEEE Trans. Plasma Sci. 40, 1977 (2012). 